\title{
Unilateral blindness due to retrobulbar hematoma after lower blepharoplasty
}

\author{
Da Woon Lee, Seok Won Hong, \\ Jun Hyuk Kim \\ Department of Plastic and Reconstructive \\ Surgery, Soonchunhyang University \\ Cheonan Hospital, Soonchunhyang \\ University College of Medicine, Cheonan, \\ Korea
}

This work was supported by the Soonchunhyang University Research Fund.
Blepharoplasty is one of the most popular cosmetic surgical procedures for people who are concerned with minimizing the effects of aging and maintaining an aesthetically attractive appearance. If periorbital surgery is not performed by an expert, the risk of complications increases. In particular, retrobulbar hematoma, which is the most serious complication after blepharoplasty, can lead to permanent blindness. We report a rare case of unilateral permanent blindness due to careless and unprofessional treatment following a retrobulbar hematoma after lower blepharoplasty. In conclusion, it is necessary to check for symptoms and signs including pain, proptosis, visual acuity, and light reflex after the operation. Careful instructions should then be given to patients and their caregivers to avoid actions that may cause postoperative bleeding. We emphasize that if a patient complains of symptoms, painkillers should not be used and computed tomography should be performed for an accurate and rapid diagnosis. Appropriate procedures must then be taken to prevent permanent vision loss.

Keywords Blepharoplasty / Blindness / Cosmetics / Retrobulbar hemorrhage

\section{INTRODUCTION}

Blepharoplasty is one of the most popular cosmetic surgical procedures for people who want to maintain a youthful appearance. Compared to other cosmetic procedures such as augmentation mammoplasty, blepharoplasty can be misunderstood as simple by physicians and patients because relatively little time is required for surgery and the technique is simpler. Therefore, it is easy to find hospitals and clinics that perform blepharoplasty.

The orbital and periorbital areas consist of important and complex structures, including various nerves and vessels. Therefore, specialized knowledge of periorbital anatomy is necessary to per-

Received: Apr 17, 2019 Revised: Apr 23, 2019 Accepted: Apr 26, 2019 Correspondence: Jun Hyuk Kim Department of Plastic and Reconstructive Surgery, Soonchunhyang University Cheonan Hospital, Soonchunhyang University College of Medicine, 31 Suncheonhyang 6-gil, Dongnam-gu, Cheonan 31151, Korea

Tel: +82-41-570-2195, Fax: +82-41-574-6133, E-mail: psdoctor@schmc.ac.kr Copyright @ 2019 The Korean Society for Aesthetic Plastic Surgery.

This is an Open Access article distributed under the terms of the Creative Commons Attribution Non-Commercial License (http://creativecommons.org/licenses/by-nc/4.0/) which permits unrestricted non-commercial use, distribution, and reproduction in any medium, provided the original work is properly cited. www.e-aaps.org form blepharoplasty. Without expert knowledge of this delicate procedure, the risk of complications after periorbital surgery, such as inflammation and postoperative bleeding, increases significantly. In particular, retrobulbar hematoma, which is the most serious complication after blepharoplasty, can lead to permanent blindness [1]. Retrobulbar hematoma may occur after blepharoplasty, surgery for orbital wall fracture, periorbital trauma, and periorbital anesthesia. According to a recent report, the incidence of retrobulbar hematoma is $0.055 \%$ and the resulting rate of permanent blindness is about $0.005 \%[1,2]$.

Here, we report a rare case of unilateral permanent blindness due to careless and unprofessional treatment following a retrobulbar hematoma after lower blepharoplasty. A review of the literature accompanies the case report.

\section{CASE REPORT}

A 63-year-old female patient was admitted to our outpatient department with severe bilateral swelling and loss of vision on the right side after a lower lid blepharoplasty at a local clinic that was not a plastic surgery center. The patient had no surgical history of periorbital area problems and had a medical history of hypertension. She was taking aspirin for hypertension. After completion of 
lower lid blepharoplasty at the local clinic, the patient complained of pressure and pain in both periorbital areas. However, the operator applied gauze compression for an hour and gave her an oral painkiller prescription, instead of conducting a thorough examination. The pain at the surgical site was somewhat relieved after taking the painkiller, but the patient's visual acuity was not adequately tested because spontaneous eye opening was not possible due to postoperative swelling.

A physical examination revealed subconjunctival hemorrhage and proptosis of both eyes and moderate or more swelling of the lower eyelid caused by the hematoma. In the left eye, exponential measurement was possible at a distance of $30 \mathrm{~cm}$. The right eye showed completely impaired vision and the pupil was fixed, with 7-mm dilation (Fig. 1).

The patient underwent immediate computed tomography (CT) with a strong suspicion of retrobulbar hematoma. On the CT imaging, a retrobulbar hematoma with anterior transposition of both orbits was observed. The hematoma in the right eye was more se-

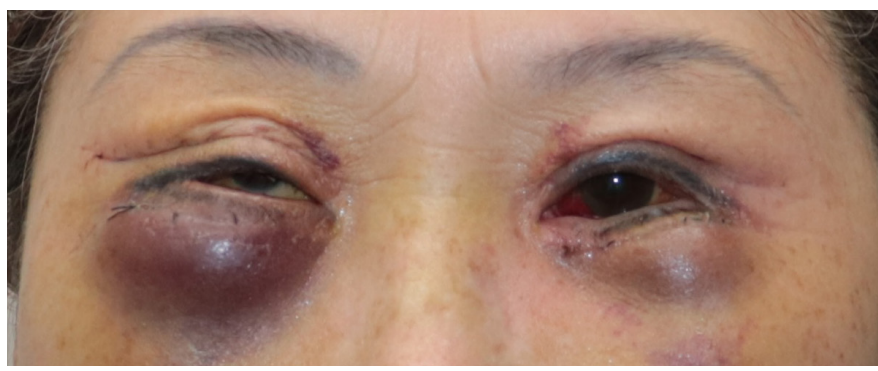

Fig. 1. Preoperative image, showing subconjunctival hemorrhage and proptosis of both eyes and moderate or more swelling of the lower eyelid. The right eye showed completely impaired vision and the pupil was fixed, with 7-mm dilation. vere and was compressing the eyeball (Fig. 2). We performed surgery to remove the hematoma and provide orbital decompression. In the intraoperative field, there were old blood clots, similar to hematomas, formed at the orbicularis oculi muscle of both periorbital areas. The orbital septal fat was hard due to fat necrosis and bleeding. Unusually, the right eye had been irregularly dissected from the periosteum of the orbital floor beyond the arcus marginalis, and a hematoma had formed under the periosteum of the orbital floor, physically compressing the orbit (Fig. 3). The hematoma was removed during surgery and no active bleeding points were found after massive saline irrigation and meticulous hemostasis. On follow-up CT imaging, we confirmed that the hematoma had been removed (Fig. 4).

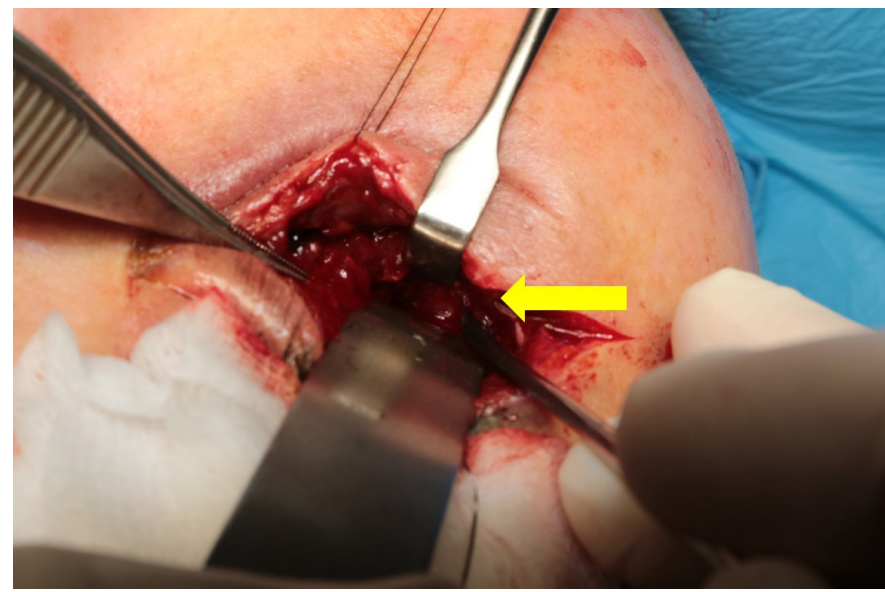

Fig. 3. Intraoperative image: the right eye was irregularly dissected from the periosteum of the orbital floor beyond the arcus marginalis and a hematoma (yellow arrow) had formed under the periosteum of the orbital floor, which physically compressed the orbit.
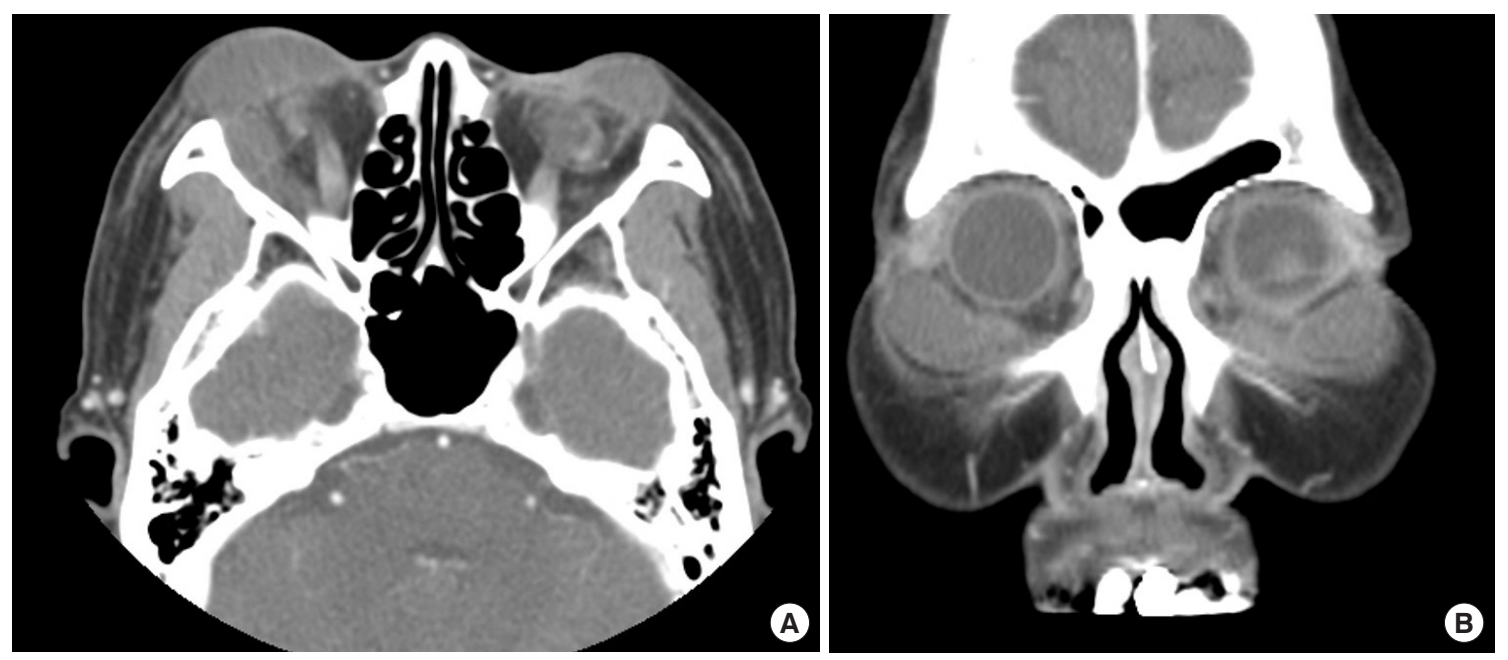

Fig. 2. Preoperative computed tomography images: a retrobulbar hematoma with anterior transposition of both orbits was observed. (A) Axial view and (B) coronal view. 

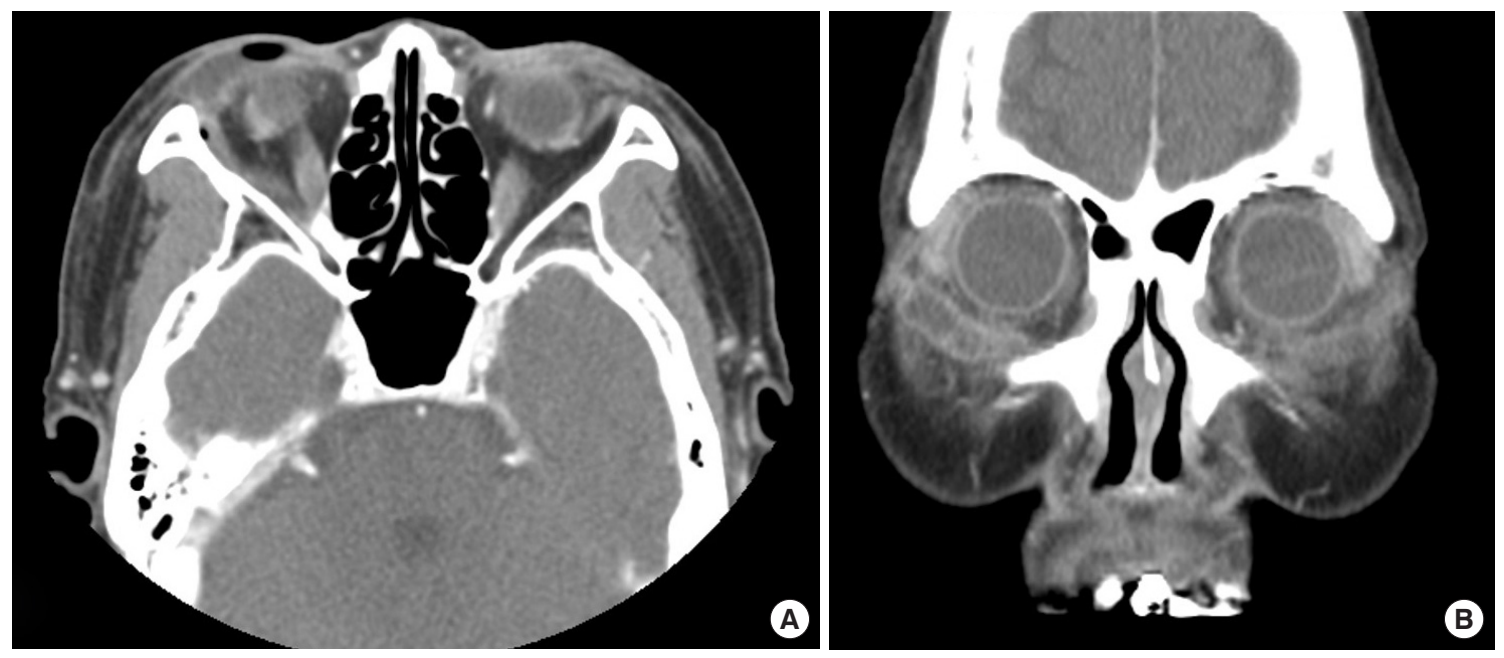

Fig. 4. Postoperative computed tomography images: the retrobulbar hematomas were removed. (A) Axial view and (B) coronal view.

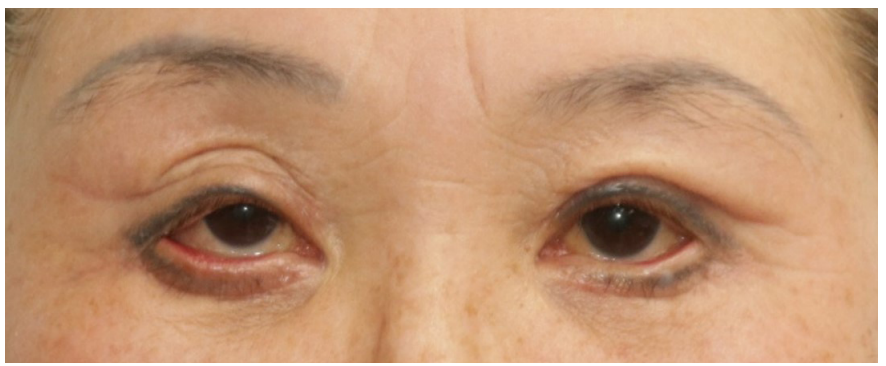

Fig. 5. Two-month postoperative image: no surgical complications were observed.

In close consultation with the department of ophthalmology, when the patient was admitted to department of plastic and reconstructive surgery, she was immediately treated with systemic steroid pulse therapy (250 $\mathrm{mg}$ of methylprednisolone each 6 hours). This treatment was maintained for 5 days postoperatively, and then was changed to oral steroid therapy (32 mg methylprednisolone), with a gradually decreasing dose.

One month after the operation, the patient began periodic outpatient follow-up visits and permanent blindness of the right eye was observed. In the left eye, the visual acuity was 0.4 at the last visit (Fig. 5).

\section{DISCUSSION}

An unprofessional approach to lower lid blepharoplasty can lead to many complications, including inflammation, ectropion, and hematoma. Retrobulbar hematoma is a rare complication that can lead to permanent vision loss. According to a report by Hass et al. [1] in 2004, the incidence of retrobulbar hemorrhage after eyelid surgery was $0.05 \%$, and $0.0045 \%$ of patients reported blindness due to postoperative bleeding. Christie et al. [3] reported that the ma- jority of cases of postoperative retrobulbar hematoma occurred after surgery for orbital wall fractures. Retrobulbar hematoma is rare and traumatic for affected patients, but careful preoperative and postoperative evaluations and management can prevent retrobulbar hematoma in most cases [4].

The pathophysiological mechanism of retrobulbar hemorrhage has not been elucidated. One theory is that excessive pulling forces used during the removal of fat cause damage to the posterior orbital vessel, resulting in hemorrhage at a remote part of the surgical site [5]. In addition, retrobulbar hematoma may occur if there is persistent bleeding in the orbicularis oculi muscle, or if adequate hemostasis of the resected fat tissue is not achieved [1,5]. According to Patrocinio et al. [6], hematoma following blepharoplasty can be classified as pre-septal or post-septal. Pre-septal hematoma, which is confined to the eyelid alone, confers no risk of impaired vision, whereas post-septal hematoma occurs in the intraocular area, causing a pressure increase in the orbit and compromising the optical nerve. These two types of hematoma can be seen in this case. Post-septal hematoma was exemplified by the irregular dissection of the right eye from the periosteum of the orbital floor beyond the arcus marginalis, and it was found that a hematoma had formed under the periosteum of the orbital floor, which physically compressed the orbit. A hematoma had also formed in the left eye, but it was a pre-septal hematoma that did not affect intraocular pressure. Generally, it has been suggested in various reports that the causes of retrobulbar hematoma remain speculative, and it is often difficult to ascertain clear reasons [3,4,7]. In contrast, in our case, we were able to ascertain the direct reason of the retrobulbar hematoma based on intraoperative findings. This case occurred because the previous doctor operated with unprofessional knowledge and skills, resulting in an abnormal dissection from the periosteum of the orbital floor during the lower blepharoplasty procedure. 
The risk of developing retrobulbar hemorrhage is increased in people who suffer from systemic diseases with bleeding tendencies, take medications increasing hemorrhagic conditions, and have a previous surgical history around the eye. The risk also increases when performing activities that increase intraocular pressure. If retrobulbar hematoma develops, the intraocular pressure increases. This can cause retinal ischemia, leading to blindness $[5,8]$. Therefore, it is necessary to evaluate the patient's preoperative comorbidities and medications to explain preoperative and postoperative precautions sufficiently.

In this case, the patient had a bleeding tendency because of her long-time use of aspirin, and she did not experience typical symptoms, such as severe unbearable pain. The pain was somewhat alleviated with oral medication. Therefore, when the patient presented with atypical postoperative symptoms, such as periorbital pain and severe headache, the possibility of retrobulbar hemorrhage should have been considered and immediate and appropriate management started. These actions could have prevented permanent blindness.

When retrobulbar hemorrhage or hematoma occurs, most patients complain of severe pain and pressure at the surgical site. Occasionally, symptoms associated with increased intraocular pressure, such as decreased visual acuity, diplopia, and nausea, may be encountered. If these symptoms are present, retrobulbar hematoma should be suspected and confirmed by CT.

If retrobulbar hematoma is diagnosed, appropriate treatment should be determined. When changes in visual acuity are observed, steroid pulse therapy or surgical decompression should be prioritized, and a decompression procedure must be performed within 24 hours to prevent vision loss [2,9]. The previous operative site should be reopened to remove the hematoma, to find the bleeding point, and to achieve hemostasis. If there is no response to these treatments, lateral canthotomy should be performed. In severe cases, relaxation of the upper and lower limb of the lateral canthal tendon should also be considered [10].

In this case, the unusual periosteum dissection of the orbital floor of the right eye appeared to have been the direct cause of the unilateral blindness. The erroneous dissection of the periosteal plane resulted in hemorrhage, leading to direct compression of the orbit and optic nerve, which caused a terrible consequence for the patient, blindness. Despite the patient's moderate pain after surgery, the doctor who operated on the patient only gave her painkillers for her symptoms. It is regrettable that the doctor missed the opportunity to take appropriate procedures because painkillers masked her symptoms.

Therefore, it is necessary to confirm symptoms and signs, such as pain, proptosis, visual acuity, and light reflex, after the operation. Careful instructions should then be given to patients and their caregivers about actions that may cause postoperative bleeding. Once again, we emphasize that if a patient complains of symptoms, pain- killers should not be used. Instead, CT should be performed to make an accurate and rapid diagnosis, then appropriate procedures should be undertaken to prevent permanent vision loss.

\section{NOTES}

\section{Conflict of interest}

No potential conflict of interest relevant to this article was reported.

\section{Ethical approval}

The study was performed in accordance with the principles of the Declaration of Helsinki.

\section{Patient consent}

The patient provided written informed consent for the publication and the use of her images.

\section{ORCID}

Da Woon Lee

Seok Won Hong

Jun Hyuk Kim

\section{REFERENCES}

1. Hass AN, Penne RB, Stefanyszyn MA, et al. Incidence of postblepharoplasty orbital hemorrhage and associated visual loss. Ophthalmic Plast Reconstr Surg 2004;20:426-32.

2. Lelli GJ Jr, Lisman RD. Blepharoplasty complications. Plast Reconstr Surg 2010;125:1007-17.

3. Christie B, Block L, Ma Y, et al. Retrobulbar hematoma: a systematic review of factors related to outcomes. J Plast Reconstr Aesthet Surg 2018;71:155-61.

4. Son KM, Park CW, Cheon JS. A case report of bilateral retrobulbar hemorrhage after lower blepharoplasty. Arch Craniofac Surg 2013;14: 133-6.

5. Lisman RD, Hyde K, Smith B. Complications of blepharoplasty. Clin Plast Surg 1988;15:309-35.

6. Patrocinio TG, Loredo BA, Arevalo CE, et al. Complications in blepharoplasty: how to avoid and manage them. Braz J Otorhinolaryngol 2011;77:322-7.

7. Goldberg RA, Marmor MF, Shorr N, et al. Blindness following blepharoplasty: two case reports, and a discussion of management. Ophthalmic Surg 1990;21:85-9.

8. Teng CC, Reddy S, Wong JJ, et al. Retrobulbar hemorrhage nine days after cosmetic blepharoplasty resulting in permanent visual loss. Ophthalmic Plast Reconstr Surg 2006;22:388-9.

9. Girotto JA, Gamble WB, Robertson B, et al. Blindness after reduction of facial fractures. Plast Reconstr Surg 1998;102:1821-34.

10. Wolfort FG, Vaughan TE, Wolfort SF, et al. Retrobulbar hematoma and blepharoplasty. Plast Reconstr Surg 1999;104:2154-62. 